\title{
Twice-Daily Theta Burst Stimulation of the Dorsolateral Prefrontal Cortex Reduces Methamphetamine Craving: A Pilot Study
}

\section{Di Zhao ${ }^{1+}$, Yongqiang $\mathrm{Li}^{2+}$, Ting $\mathrm{Liu}^{3}$, Valerie Voon ${ }^{4 *}$ and Ti-Fei Yuan ${ }^{1,5 *}$ \\ ' Shanghai Key Laboratory of Psychotic Disorders, Shanghai Mental Health Center, Shanghai Jiao Tong University School of Medicine, Shanghai, China, ${ }^{2}$ Rehabilitation Medicine Center, The First Affiliated Hospital of Nanjing Medical University, Nanjing, China, ${ }^{3}$ School of Psychology, Nanjing Normal University, Nanjing, China, ${ }^{4}$ Department of Psychiatry, University of Cambridge, Cambridge, United Kingdom, ${ }^{5}$ Co-innovation Center of Neuroregeneration, Nantong University, Nantong, China}

Objectives: Transcranial magnetic stimulation (TMS) holds potential promise as a therapeutic modality for disorders of addiction. Our previous findings indicate that high-frequency repetitive transcranial magnetic stimulation (rTMS) over the left dorsallateral prefrontal cortex (DLPFC) and low-frequency rTMS over the right DLPFC can reduce drug craving for methamphetamine. One major issue with rTMS is the duration of treatment and hence potential dropout rate. Theta burst stimulation (TBS) has been recently shown to be non-inferior relative to repetitive transcranial magnetic stimulation for major depression. Here, we aim to compare the clinical efficacy and tolerability of intermittent and continuous theta burst stimulation protocols targeting left or right dorsolateral prefrontal cortex on methamphetamine craving in abstinentdependent subjects.

Methods: In this randomized single-blind pilot study, 83 abstinent methamphetaminedependent subjects from a long-term residential treatment program were randomly allocated into three groups: intermittent theta burst stimulation (iTBS) over the left DLPFC (active group), continuous theta burst stimulation (cTBS) over the left DLPFC (active control group), or cTBS over the right DLPFC (active group) was administered twice daily over 5 days for a total of 10 sessions. We measured the primary outcome of cue-induced craving and secondarily sleep quality, depression, anxiety, impulsivity scores, and adverse effects.

Results: We show a pre- vs. postintervention effect on craving, which, on paired $t$ tests, showed that the effect was driven by iTBS of the left DLPFC and cTBS of the right DLPFC, reducing cue-induced craving but not cTBS of the left DLPFC. We did not show the critical group-by-time interaction. The secondary outcomes of depression, anxiety, and sleep were unrelated to the improvement in craving in the left iTBS and right cTBS group. In the first two sessions, self-reported adverse effects were higher with left iTBS when compared to right cTBS. The distribution of craving change suggested greater clinical response (50\% improvement) with right cTBS and a bimodal pattern of effect with left iTBS, suggesting high interindividual variable response in the latter. 
Conclusion: Accelerated twice-daily TBS appears feasible and tolerable at modulating craving and mood changes in abstinent methamphetamine dependence critically while reducing session length. We emphasize the need for a larger randomized controlled trial study with a sham control to confirm these findings and longer duration of clinically relevant follow-up.

\section{Clinical Trial Registration: Chinese Clinical Trial Registry number, 17013610.}

Keywords: addiction, transcranial magnetic stimulation, theta burst stimulation, craving, DLPFC (dorsolateral prefrontal cortex)

\section{INTRODUCTION}

Disorders of addiction, or compulsive drug-seeking behaviors despite adverse negative consequences, are characterized by abnormal brain network function (Goldstein and Volkow, 2011; Everitt and Robbins, 2016). Preclinical and translational studies highlight a prominent role for hypoactivity of the prefrontal cortex (PFC) with chronic stimulant exposure, leading to the hypothesis that potentiation of PFC function with brain stimulation might improve addiction management (Diana et al., 2017). In the recent decade, non-invasive, repetitive transcranial magnetic stimulation (rTMS) over the dorsolateral prefrontal cortex (DLPFC) has been used to treat cue-induced craving or drug intake across different types of drug dependence, including methamphetamine, cocaine, and heroin (Shen et al., 2016; Terraneo et al., 2016; Su et al., 2017). A range of prefrontal neural regions have been targeted with rTMS including superior frontal gyrus (Rose et al., 2011) or medial prefrontal cortex (Hanlon et al., 2017), whereas we focus here on DLPFC targeting. Convergent evidence has suggested that facilitating the left DLPFC or inhibiting the right DLPFC may reduce craving and substance consumption in patients with substance dependence (Zhang et al., 2019). High-frequency excitatory rTMS of the left DLPFC has been reported to be effective in cocaine use disorder [e.g., $15 \mathrm{~Hz} / 8$ sessions/100\% motor threshold (Terraneo et al., 2016), $15 \mathrm{~Hz} / 10$ sessions/100\% motor threshold (Politi et al., 2008), and $10 \mathrm{~Hz} /$ single session/90\% motor threshold (Camprodon et al., 2007)] and nicotine use disorder [e.g., high frequency/13 session/120\% motor threshold, deep TMS over bilateral lateral prefrontal and insula (Dinur-Klein et al., 2014), $10 \mathrm{~Hz} / 10$ sessions/100\% motor threshold, and $20 \mathrm{~Hz} / 8$ sessions/110\% over the DLPFC (Amiaz et al., 2009; Sheffer et al., 2018)]. Other stimulants such as methamphetamine craving similarly decreased with high-frequency left DLPFC rTMS (10 Hz/5 sessions/80\% motor threshold) (Su et al., 2017), but with enhanced cue craving observed with low frequency $(1 \mathrm{~Hz} /$ single session/100\% motor threshold) (Li et al., 2013b). In heroin-dependent subjects, highfrequency rTMS of the left DLPFC similarly decreased craving (10 Hz/5 sessions/100\% motor threshold) (Shen et al., 2016). In contrast, alcohol-dependent subjects showed a different response as a function of laterality with decreased craving with highfrequency rTMS of the right DLPFC (10 Hz/10 sessions/110\% motor threshold) (Mishra et al., 2010), with no effects on craving in female alcoholics with high-frequency rTMS of the left DLPFC (20 Hz/10 sessions/110\% motor threshold) (Höppner et al., 2011).
The rTMS protocol is commonly administered for up to 10$30 \mathrm{~min} /$ day with treatment duration lasting between 20 and 30 days. Critically, as treatment compliance is a major issue in drug addiction, decreasing the duration of treatment might enhance the likelihood of completed treatment. Here, we focus on shorter stimulation protocols to reduce session lengths and visits that might lead to improved accessibility for non-invasive neuromodulation for addiction management.

Intermittent or continuous theta burst stimulation (iTBS or cTBS) are TMS protocols that have been shown to, respectively, enhance or inhibit local brain regional activity with long-lasting effect (Huang et al., 2005; Suppa et al., 2016). The protocols involve 600 pulses and requires $3 \mathrm{~min}$ for iTBS and $40 \mathrm{~s}$ for cTBS (Huang et al., 2005). Previous studies have demonstrated that iTBS has shown comparable neurophysiological excitatory effects to $10 \mathrm{~Hz}$ rTMS (Di Lazzaro et al., 2005; Lopez-Alonso et al., 2014). Continuous TBS for 10 sessions to the medial prefrontal cortex has shown potential efficacy for cocaine use disorder (Hanlon et al., 2017). Recently, iTBS was shown in a randomized trial of major depression to be non-inferior to the $10 \mathrm{~Hz}$ rTMS protocol in reducing depressive symptoms with similar tolerability and safety profiles (Blumberger et al., 2018). Moreover, iTBS showed similar efficacy to $10 \mathrm{~Hz}$ rTMS but, given its shorter duration, might allow a 10-fold increase in the number of patients treated in cocaine use disorder (Sanna et al., 2019). Preliminary studies in major depression have also reported that twice-daily rTMS appears feasible, tolerable, and capable of achieving efficacy similar to once-daily rTMS while reducing treatment course length twofold (McGirr et al., 2015; Modirrousta et al., 2018; Schulze et al., 2018). A recent study has also shown accelerated iTBS as a treatment for cocaine use disorder (Steele et al., 2019). However, no trials have been published to date that explore the feasibility and clinical effects achieved with accelerated (twice-daily) TBS approaches in methamphetamine-dependent patients.

A recent meta-analysis has supported the different left/right hemispheric roles for craving (e.g., cued craving is associated with left DLPFC) and impulsivity (e.g., the suppression of right DLPFC increases the level of impulsive decision making) (Gordon, 2016). Previous studies have suggested that potentiation of the left DLPFC and suppression of the right DLPFC may be effective in reducing cue-induced craving ( $\mathrm{Li}$ et al., 2013a; Shen et al., 2016; Terraneo et al., 2016; Yavari et al., 2016; Diana et al., 2017). Furthermore, iTBS to the left DLPFC has been shown to produce transsynaptic suppression 
of the right DLPFC (i.e., the dominant hemisphere in righthanded individuals) via transcallosal connections (George et al., 1999). In the present study, the rationale for choosing iTBS over the left DLPFC and cTBS over the right DLPFC is supported by the above-mentioned studies. We hypothesized that iTBSL DLPFC and cTBS-R DLPFC would demonstrate efficacy in improving craving symptoms and that cTBS-L DLPFC might act as an active control with an increase in craving symptoms. We further included other secondary outcome measures to assess the role of potential confounders given the known effects of neuromodulation of the DLPFC on mood and impulsivity measures. Critically, we hypothesized that a twice-daily TBS would be feasible in methamphetamine use disorder. We further compared tolerability and self-reported adverse events across different sessions of treatment and among the three accelerated TBS protocols.

\section{MATERIALS AND METHODS}

\section{Human Subjects}

All the participants were right-handed male, 18-60 years old, and recruited from a long-term residential treatment center. Inclusion criteria included those whose main diagnosis was methamphetamine use disorder with a duration of at least 1 year and using more than $0.1 \mathrm{~g}$ a day for at least 3 months. Subjects had a positive urine drug screening test upon admission to a long-term residential treatment program. Subjects could use other substances before admission but must have had only methamphetamine use disorder as their primary addiction diagnosis (except nicotine use disorder). The diagnosis of moderate-severe methamphetamine use disorder was confirmed by a senior psychiatrist [Diagnostic and Statistical Manual of Mental Disorder, Version V (DSM-V)]. The psychiatrist ruled out other severe psychiatric disorders including schizophrenia, bipolar disorder, or severe major depression. Exclusion criteria included a history of other psychiatric disorders, epilepsy, cardiovascular complications, and other contraindications to TMS (e.g., metal implants in the skull). Subject characteristics and previous methamphetamine use history is reported in Table 1. In the rehabilitation center, all participants received standardized rehabilitation including daily physical exercise, supportive therapy on relapse prevention, but no medications. As the rehabilitation program is an enforced residential drug treatment program, participants maintained abstinence in the study. Ethics approval was granted by the Research Ethics Boards of Shanghai Mental Health Center, Nanjing Normal University and the local safety monitoring board (Chinese Clinical Trial Registry number, 17013610). All participants provided written informed consent in accordance with the Declaration of Helsinki.

A total of 83 inpatients were recruited and randomly assigned (with a computer generated number sequence) into iTBS-L DLPFC $(n=27)$, cTBS-L DLPFC $(n=26)$, and cTBS-R DLPFC $(n=30)$ groups. All patients were naive to TMS. Patients recruited in the study did not participate in other intervention studies before. All patients received twice-daily TBS over five consecutive days for a total of 10 sessions. The following were not included in the data set: six subjects were transferred to a different rehabilitation center before study onset (one iTBS$\mathrm{L}$ and five cTBS-L), and three subjects withdrew before study completion (three cTBS-L). There were no significant differences in demographic variables (e.g., age, years of drug abuse history, number of cigarettes smoked per day, monthly dosage, interval between admission into the rehabilitation center and entry into the study, baseline craving, sleep quality, depression, anxiety, and impulsivity) between study completers and non-completers.

\section{DLPFC-TBS Procedures}

TBS was applied with a CCY-I TMS instrument (Yiruide Co., Wuhan, China), using a figure eight or round-shaped coil for targeted stimulation over the left or right DLPFC. The TMS intensity for each individual participant was calculated as $70 \%$ of the resting motor threshold. The motor hand area was localized by TMS that evoked responses of the contralateral abductor pollicis brevis (APB) muscle. The resting motor threshold was determined as the TMS intensity that elicited the APB muscle responses in 5 out of 10 TMS pulses, which produced five motor-evoked potentials responses of at least $50 \mathrm{mV}$ in 10 trials (Kammer et al., 2001) (iTBS-L DLPFC, $28 \% \pm 6 \%$; cTBSL DLPFC, $27 \% \pm 6 \%$; cTBS-R DLPFC, $29 \% \pm 7 \%$ ). The DLPFC target was located using the Yiruide TMS Location Cap based on the 10-20 electroencephalography (EEG) system [i.e., F3 and F4 localization for the left and right DLPFC, respectively (Herwig et al., 2003)]. The TMS coil was held above the head of participants with a customized coil holder, and the handle of the coil was rotated to a position where the plane of the coil made an angle of $45^{\circ}$ relative to the midline, producing a posterior-anterior current flow within underlying cortical areas. The procedure used for iTBS is composed of three pulse trains of $50 \mathrm{~Hz}$ at $70 \%$ resting motor threshold (MT) (based on pilot study and the tolerance level for most subjects), which was repeated at $5 \mathrm{~Hz}$ ( $2 \mathrm{~s}$ on, 8 -s interval) for $3 \mathrm{~min}$ (600 pulses in total). In the case of cTBS, three pulse trains of $50 \mathrm{~Hz}$ at $70 \%$ resting MT was repeated at $200 \mathrm{~ms}$ for $40 \mathrm{~s}$ (600 pulses in total). The interval of time between the two sessions of treatment delivered on the same day was $\sim 4 \mathrm{~h}$. Baseline craving, quality of sleep, depression, anxiety, and impulsivity were assessed before the first TBS session (pre-TBS) and, on day 6, the day after the final TBS session (post-TBS).

\section{Blinding}

In this randomized, single-blind study, one experimenter (who administered the intervention) was not blinded to the group assignment, while both the participants and another experimenter (the outcome assessor) were blinded. After all treatments, we asked them to guess whether they had received effective or non-effective stimulation and how much they felt stimulation may have affected them [1 (much worse) to 9 (much better)] to monitor effectiveness of the blinding.

\section{Measurement}

The main outcome measure was the craving score evaluation, which was performed as previously described (Shen et al., 2016). 
TABLE 1 | Demographic and clinical characteristics of patients.

\begin{tabular}{|c|c|c|c|c|c|}
\hline & iTBS-L-D ( $n=26)$ & cTBS-L-D $(n=18)$ & cTBS-R-D ( $n=30)$ & $\boldsymbol{F}$ & $P$ value \\
\hline Age (years) & $31.30(9.60)$ & $29.50(5.50)$ & $28.23(6.24)$ & 1.19 & 0.31 \\
\hline Education (years) & $8.54(2.45)$ & $9.50(1.90)$ & $8.18(1.95)$ & 2.16 & 0.12 \\
\hline Number of cigarettes smoked/day & $8.88(6.10)$ & $7.33(2.4)$ & $8.20(6.08)$ & 0.44 & 0.65 \\
\hline Duration of meth use (years) & $6.50(3.71)$ & $5.78(3.39)$ & $6.80(3.29)$ & 0.49 & 0.61 \\
\hline Duration of current abstinence (months) & $6.80(5.20)$ & $7.89(6.70)$ & $5.43(4.20)$ & 1.29 & 0.28 \\
\hline Meth use before abstinence ( $\mathrm{g} / \mathrm{month})$ & $18.80(8.89)$ & $23.88(7.96)$ & $22.48(14.03)$ & 1.47 & 0.24 \\
\hline $\begin{array}{l}\text { Interval between admission into the } \\
\text { rehabilitation center and entry into the } \\
\text { study (days) }\end{array}$ & 82.35 (62.99) & $101.28(59.48)$ & $99.37(71.80)$ & 0.615 & 0.54 \\
\hline Baseline Craving & $65.19(22.20)$ & $65.83(22.44)$ & $74.83(19.14)$ & 1.77 & 0.18 \\
\hline Baseline PSQI & $6.7(3.1)$ & $7.1(3.1)$ & $8(2.7)$ & 1.41 & 0.25 \\
\hline Baseline BDI & $13.6(7.5)$ & $12.8(7.2)$ & $17.5(9.4)$ & 2.405 & 0.1 \\
\hline Baseline BAI & $25(4.5)$ & $29.1(9.98)$ & $29.8(8.83)$ & 2.79 & 0.07 \\
\hline Baseline BIS-11 & $80.36(14.43)$ & $87.22(14.82)$ & $83.67(14.07)$ & 1.226 & 0.30 \\
\hline
\end{tabular}

Data are given as mean (SD). BDI, Beck Depression Inventory; BAI, Beck Anxiety Inventory; BIS-11, Barratt Impulsiveness Scale 11.

The patients watched a video showing methamphetamine intake for $5 \mathrm{~min}$ followed by a visual analogue scale (VAS) to evaluate cue-induced craving scores [range: 0 (no desire or wanting) to 100 (very high desire or wanting)]. The same video was used before treatment (pre-TBS) and after 5 days of treatment (post-TBS). Patients were assessed for cued craving score pre- and posttreatment, which was used to categorize subjects as responders or non-responders. For major depression, changes from baseline values were examined for the 17-item Hamilton Rating Scale in two subject groups (responders and non-responders) (Bakker et al., 2015). Response to TMS was defined as 50\% symptoms reduction from pre- to posttreatment (Schulze et al., 2018). Similarly, in the present study, a TBS responder was defined as having at least a $50 \%$ reduction in cued craving scores post-TBS compared with baseline.

As the mechanism underlying neuromodulation effects targeting the DLPFC may be related to effects on other symptoms, most particularly depression and impulsivity, we also assessed other secondary outcome measures. The 21item Beck Depression Inventory (BDI) and 21-item Beck Anxiety Inventory (BAI) were used to assess depressive and anxiety symptoms (Beck et al., 1961; Beck et al., 1988). The Pittsburgh Sleep Quality Index (PSQI) was used to assess sleep quality and consists of 19 self-rated items with 7 components: subjective sleep quality, sleep latency, sleep duration, sleep efficiency, sleep disturbance, use of sleep medication, and daytime dysfunction (Buysse et al., 1989). The 30-item Barratt Impulsiveness Scale-11 (BIS-11) is a self-report measure of impulsivity that assesses six different subtypes of impulsivity (attention, motor, self-control, cognitive complexity, perseverance, and cognitive instability impulsiveness) (Reise et al., 2013).

Subjects were assessed for nine adverse reactions after each treatment session including headache, neck pain, scalp pain, tingling, itching, burning sensations, sleepiness, trouble concentrating, and acute mood change. Each item was scored on a scale of 1 (mild)-10 (severe), with the total score recorded as the sum of all nine items. For tolerability comparisons, each patient's mean self-reported total score across all sessions was calculated.

\section{Statistical Analysis}

The data were assessed for normality of distribution (Kolmogorov-Smirnov test) and outliers. For data that were not normally distributed, non-parametric statistical analyses were used. There were no outliers. Homogeneity of our intervention groups for baseline demographic and clinical characteristics was confirmed. One-way analyses of variance (ANOVAs) or chi-square test were used to compare group differences for continuous or dichotomous variable comparisons, respectively. A two-way repeated measures analysis of variance (RMANOVA) was used to analyze the effects of TBS on our primary outcome of cued craving and also our secondary outcomes of sleep quality, anxiety, depression, and impulsivity between groups, respectively, with time (pre, post) as a within-subject factor and group (iTBS-L DLPFC, cTBS-L DLPFC, and cTBS-R DLPFC) as a between-subjects factor. Two-sided paired $t$ tests were performed between conditions when a significant main effect or time $\times$ group interaction was observed. Multiple comparisons were corrected using false discovery rate (FDR) correction (Benjamini and Yekutieli, 2001). When we observed a significant TBS effect in craving scores and other clinical indices (sleep quality, depression, anxiety, and impulsivity), Pearson's correlation was conducted in exploratory analyses for each TBS group separately to test the relationship between the two indices. Multiple comparisons were corrected for pairwise correlations using FDR correction.

In a secondary analysis of the cued craving score, the improvement percentage was calculated as the percentage change between baseline pre- and post-TBS treatment craving scores. Kernel density estimate (KDE), a non-parametric method to estimate the probability density function of a continuous random variable, was then used to model the distribution of craving score changes acting as a continuous replacement for the discrete histogram. The Kolmogorov-Smirnov test showed that the probability distribution was not normally distributed. We then 


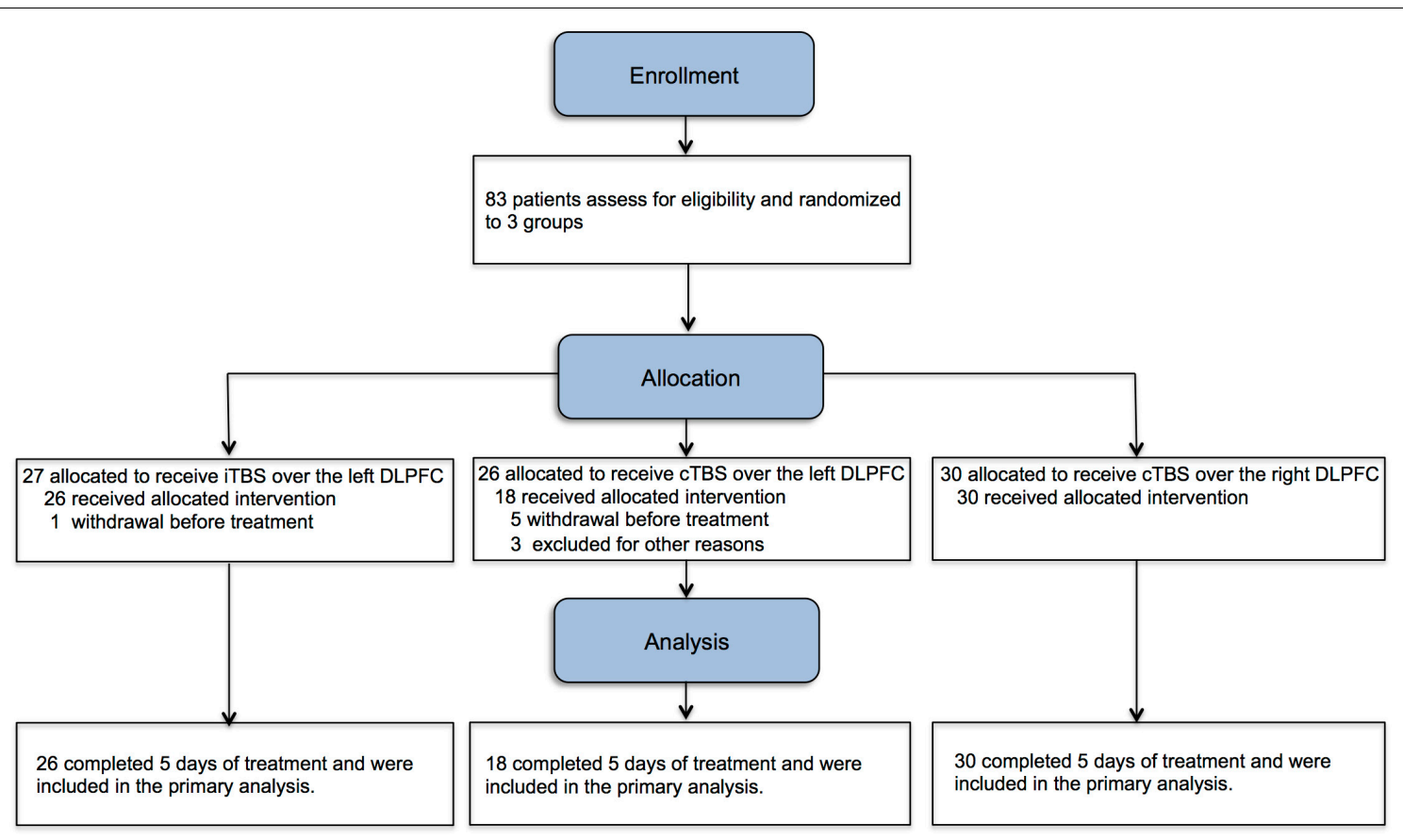

FIGURE 1 | Study flowchart. Eighty-three male methamphetamine-dependent subjects were assigned into three groups for twice-daily theta burst stimulation (TBS) procedures for continuous 5 days (10 sessions). iTBS, intermittent theta burst stimulation; cTBS, continuous theta burst stimulation.

used the non-parametric Wald-Wolfowitz test to compare the distributions between groups.

For adverse effects, the total scores for all nine items (headache, neck pain, scalp pain, tingling, itching, burning sensations, sleepiness, trouble concentrating, and acute mood change) after each treatment were calculated and compared using a two-way RMANOVA with treatment sessions (110) as a within-subject factor and group as a betweensubjects factor. The total scores for each adverse event were analyzed using one-way ANOVA. Further paired $t$ tests were all FDR corrected.

For blinding effectiveness, the self-report ratings after all treatments were compared using the Kruskal-Wallis Test with group as a factor.

All data were analyzed by IBM SPSS Statistics version 20.1 (IBM Inc., New York, NY, United States) and Matlab R2014b (MathWorks, MA, United States) environments. The statistical significance threshold was set at $P<0.05$ (two-tailed).

\section{RESULTS}

Seventy-four subjects completed 5 days of treatment (iTBSL DLPFC, $N=26$; cTBS-L DLPFC, $N=18$ and cTBS-R DLPFC, $N=30$ ) (Figure 1). There were no group differences at baseline in age, years of drug abuse history, number of cigarettes smoked per day, monthly dosage, interval between admission into the rehabilitation center and entry into the study, baseline craving, sleep quality, depression, anxiety, and impulsivity (Table 1).

\section{Effects of TBS on Craving, Sleep Quality, Mood, and Impulsivity}

Table 2 shows the results of ANOVAs conducted for cueinduced craving, sleep quality, mood, anxiety, and impulsivity. The craving score showed a significant main effect of time $\left(F_{1,73}=21.01, P<0.001, \eta^{2}=0.23\right)$, suggesting that craving

TABLE 2 | Results of the ANOVAs conducted for craving, PSQI, BDI, BAl, and BIS.

\begin{tabular}{llcccl}
\hline Measure & Source & df & $\boldsymbol{F}$ & Sig. & $\eta^{2}$ \\
\hline Cued-Craving & Time & 1 & 21.01 & $<0.001$ & 0.23 \\
& Group & 2 & 0.47 & 0.63 & 0.01 \\
\multirow{3}{*}{ PSQI } & Time $\times$ group & 2 & 2.00 & 0.14 & 0.05 \\
& Time & 1 & 38.35 & $<0.001$ & 0.35 \\
& Group & 2 & 3.68 & 0.03 & 0.09 \\
BDI & Time $\times$ group & 2 & 3.38 & 0.04 & 0.09 \\
& Time & 1 & 49.64 & $<0.001$ & 0.41 \\
& Group & 2 & 0.17 & 0.84 & 0.005 \\
BAl & Time $\times$ group & 2 & 3.05 & 0.05 & 0.08 \\
& Time & 1 & 9.06 & 0.004 & 0.11 \\
& Group & 2 & 4.05 & 0.02 & 0.10 \\
BIS & Time $\times$ group & 2 & 0.27 & 0.76 & 0.008 \\
& Time & 1 & 0.18 & 0.68 & 0.002 \\
& Group & 2 & 0.75 & 0.48 & 0.02 \\
& Time $\times$ group & 2 & 0.74 & 0.48 & 0.02 \\
\hline
\end{tabular}

Pittsburgh Sleep Quality Index (PSQI); BDI, Beck Depression Inventory; BAI, Beck Anxiety Inventory; BIS, Barratt Impulsiveness Scale. 
improved pre- vs. post-intervention. To assess the role of specific conditions, two-sided paired $t$ tests (FDR corrected) were used to compare craving scores before and after treatment. As shown in Figure 2A, iTBS-L DLPFC $(P=0.01)$ and cTBSR DLPFC $(P=0.001)$ significantly reduced craving scores but not cTBS-L DLPFC $(P=0.52)$ (Figure 2A). There were no significant main effects of group $\left(F_{2,146}=0.47, P=0.63\right.$, $\left.\eta^{2}=0.01\right)$ nor time $\times$ group interaction $\left(F_{2,146}=2, P=0.14\right.$, $\left.\eta^{2}=0.05\right)$.

KDE of the distribution functions for percentage improvement (cue-induced craving) from pretreatment for each group is shown in Figure 3. The Kolmogorov-Smirnov test indicated that the distribution of percent change in craving was not normal for iTBS-L DLPFC $(D=0.32, P=0.006)$, cTBS-L DLPFC $(D=0.37, P=0.02)$, and cTBS-R DLPFC $(D=0.33, P=0.003)$. We showed a significant difference in the probability distribution between iTBS-L DLPFC and CTBS-L DLPFC (Wald-Wolfowitz test, $P=0.03$ ) with a marginally significant difference between cTBS-L DLPFC and cTBS-R DLPFC $(P=0.06)$. In the cTBS L-DLPFC group, patients' craving symptoms in three responders (16.67\%) improved on average by 45 points (from 65 to $20 ; 95 \% \mathrm{CI},-9.1$ to 99.1 ). In contrast, craving scores in 11 responders $(36.67 \%)$ in the cTBS R-DLPFC group and 6 responders $(23.08 \%)$ in the iTBS L-DLPFC group demonstrated improvements of 57.3 (from 72.7 to $15.4 ; 95 \% \mathrm{CI}, 41.9-72.6$ ) and 55 (from 66.7 to $11.7 ; 95 \% \mathrm{CI}$, 40.5-69.5), respectively.

The sleep quality score demonstrated a main effect of time $\left(F_{1,73}=38.35, P<0.001, \eta^{2}=0.35\right)$ and group $\left(F_{2,146}=3.68\right.$, $\left.P=0.03, \eta^{2}=0.09\right)$ and a time $\times$ group interaction $\left(F_{2,146}=3.38\right.$, $\left.P=0.04, \eta^{2}=0.09\right)$. In the cTBS-L DLPFC $(P=0.009$, FDR corrected) and iTBS-L DLPFC $(P=0.0002$, FDR corrected $)$ groups, sleep quality scores showed a significant reduction or improvement with no differences shown with cTBS-R DLPFC ( $P=0.08$, FDR corrected) (Figure 2B). Thus, although sleep quality improved pre- vs. posttreatment, this was driven by the cTBS-L and iTBS-L relative to cTBS-R DLPFC groups.

Repeated measures analysis of variance for depressive symptom scores indicated a main effect of time $\left(F_{1,73}=49.64\right.$, $\left.P<0.001, \eta^{2}=0.41\right)$, suggesting an overall pre- vs. posttreatment improvement. As shown in Figure 2C, compared to baseline, there was a significant decrease in all posttreatment depression scores (iTBS-L DLPFC, $P=0.0009$; cTBS-L DLPFC, $P=0.001$; cTBS-R DLPFC, $P=0.0005$; FDR corrected). Neither a significant main effect of group $\left(F_{1,73}=0.17, P=0.84, \eta^{2}=0.005\right)$ nor time $\times$ group interaction $\left(F_{2,146}=3.05, P=0.05\right.$, $\left.\eta^{2}=0.08\right)$ was found.

Repeated measures analysis of variance for anxiety scores suggested a main effect of time $\left(F_{1,73}=9.06, P=0.004, \eta^{2}=0.11\right)$ and group $\left(F_{2,146}=4.05, P=0.02, \eta^{2}=0.1\right)$. In the post hoc analysis, only iTBS-L DLPFC showed a decrease in anxiety scores $(P=0.001$, FDR corrected) (Figure 2D). The interaction of time $\times$ group $\left(F_{2,146}=0.27, P=0.76, \eta^{2}=0.008\right)$ was not significant.

Repeated measures analysis of variance for impulsivity revealed neither a main effect nor interaction (Table 2 and Figure 2E).

\section{Correlation Analyses Between Changes Across Different Clinical Indexes}

We conducted exploratory analyses on the primary outcome measure of craving and secondary outcome measures to assess potential relationships to other clinical outcomes. A positive correlation of changes between craving and sleep quality $(r=0.56$, $P=0.045$, FDR corrected $)$ and craving and depression $(r=0.495$, $P=0.037$, FDR corrected) was observed in the cTBS-L DLPFC group but critically not in the iTBS-L DLPFC and cTBS-R DLPFC groups, both of which demonstrated significant improvement in craving with intervention. Changes between sleep quality and anxiety in cTBS-L DLPFC was significantly correlated $(r=0.519$, $P=0.003$, FDR corrected). In the cTBS-R DLPFC group, changes between depression and anxiety were also significantly correlated $(r=0.707, P=0.001$, FDR corrected) (Figure 4).

\section{Adverse Reactions}

All treatments were safe, and no seizures were reported. For the mean self-reported adverse reactions total score after each treatment, RMANOVA showed a main effect of treatment sessions $\left(F_{9,657}=13.37, P<0.001, \eta^{2}=0.47\right)$ and treatment sessions $\times$ group interaction $\left(F_{18,1,314}=3.89, P=0.001\right.$, $\left.\eta^{2}=0.27\right)$. Further analysis suggested that total score of adverse reactions in cTBS-R DLPFC was significantly lower than in iTBS-L DLPFC after the first treatment session $(P=0.01$, FDR corrected) and second treatment session $(P=0.04$, FDR corrected) (Figure 5A). As shown in Figure 5B and Table 3, the cTBS-L DLPFC group exhibited the lowest percentage (5.5\%, 17 out of 18) of adverse effects after the last treatment, and the cTBS-R DLPFC and iTBS-L group demonstrated mild adverse reactions.

\section{Blinding Effectiveness}

For the self-report ratings after all treatments, Kruskal-Wallis test displayed no significant main effect of group $(P=0.906)$.

\section{DISCUSSION}

Adherence to treatment is a major issue in disorders of addiction. Our findings indicate the possible efficacy and tolerability of accelerated twice-daily iTBS-left or cTBS-right DLPFC treatment over 5 days in reducing craving for methamphetamine but not cTBS-left DLPFC. To our knowledge, this is the first singleblind randomized trial to systematically compare the effects of accelerated TBS procedures for methamphetamine craving targeting the DLPFC. All three interventions similarly improved mood scores, and iTBS-left TBS also improved sleep and anxiety scores, as there was no relationship with craving improvements, our craving findings may be a primary effect. Our findings converge with previous observations of efficacy of high-frequency rTMS of the left DLPFC and low-frequency rTMS of the right DLPFC to modulate craving in disorders of addictions (Li et al., 2013a; Shen et al., 2016; Terraneo et al., 2016; Yavari et al., 2016; Diana et al., 2017). Studies reporting alternate outcomes may be related to difference in TMS protocols or in efficacy as a function of the substance (Mishra et al., 2010; Höppner et al., 2011). These 


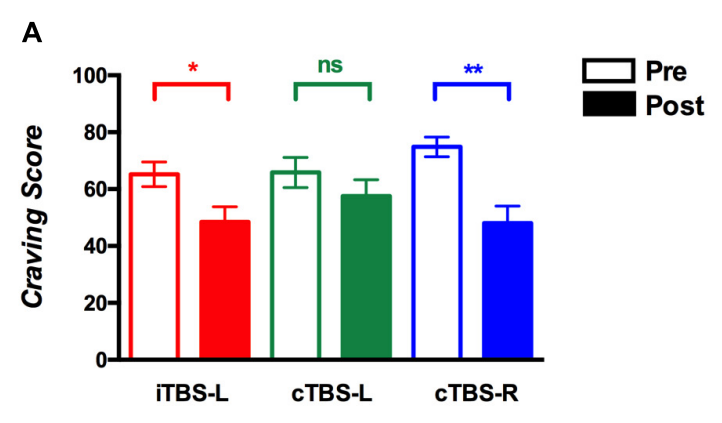

B

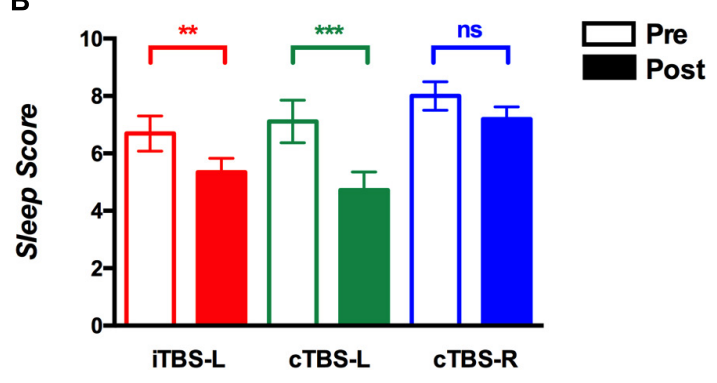

D

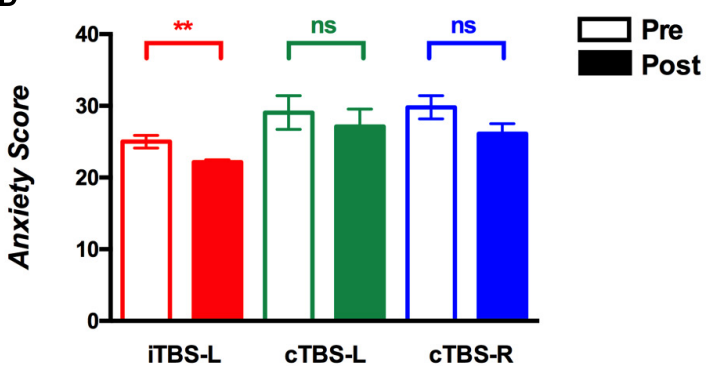

C

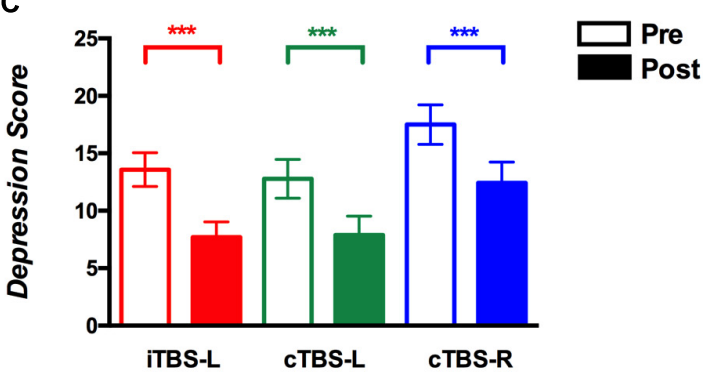

E

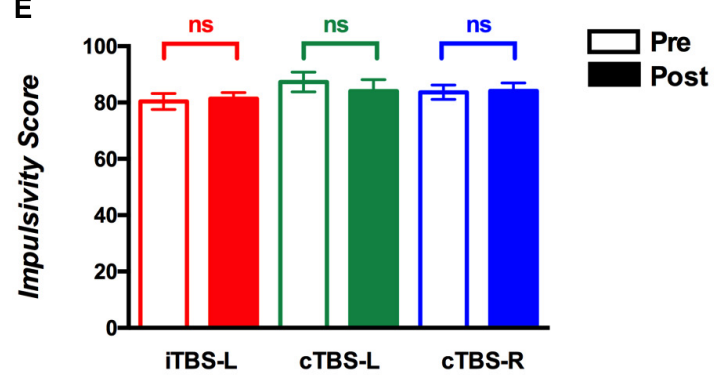

FIGURE 2 | Theta burst stimulation (TBS) intervention on cue-induced craving, quality of sleep, depression, anxiety, and impulsivity scores. The $y$-axis shows mean scores before and after treatment sessions on (A) cue-induced craving, (B) sleep quality, (C) depressive symptoms, (D) anxiety symptoms, and (E) impulsivity (red, intermittent theta burst stimulation of the left dorsolateral prefrontal cortex (iTBS-L DLPFC); green, continuous TBS of the left DLPFC (cTBS-L DLPFC); blue, continuous TBS of the right DLPFC (cTBS-R DLPFC). Multiple comparisons were corrected using false discovery rate (FDR) correction, ${ }^{*} P<0.05,{ }^{* *} P<0.01$, ${ }^{\star \star \star} P<0.001$; error bars denote SEM).

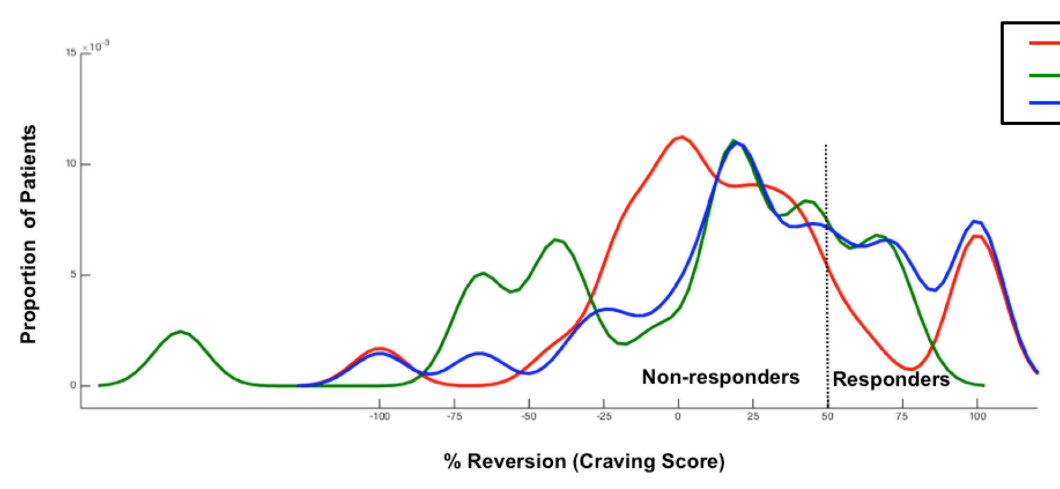

FIGURE 3 | The response distribution curve. Kernel density estimates of cued-craving distributions (shown as percentage improvement from pre- to posttreatment) in methamphetamine-dependent patients $(N=74)$ who were receiving either intermittent TBS of the left dorsolateral prefrontal cortex (iTBS-L DLPFC) (red), or continuous TBS of the left DLPFC (cTBS-L DLPFC) (green) or continuous TBS of the right DLPFC (cTBS-R DLPFC) (blue). A TBS responder was defined as having at least a 50\% reduction in cued craving scores post-TBS compared with baseline. 
A

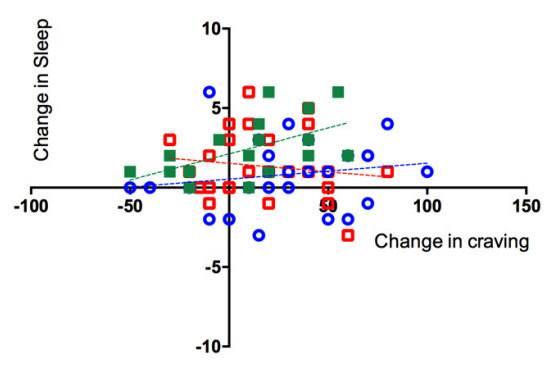

C

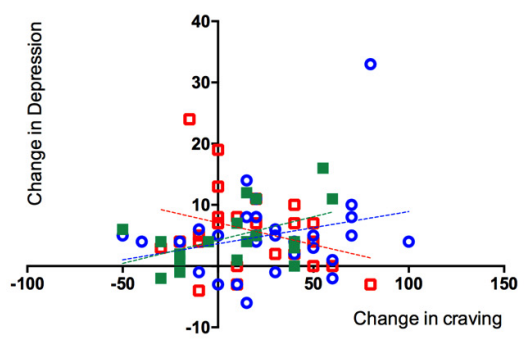

E

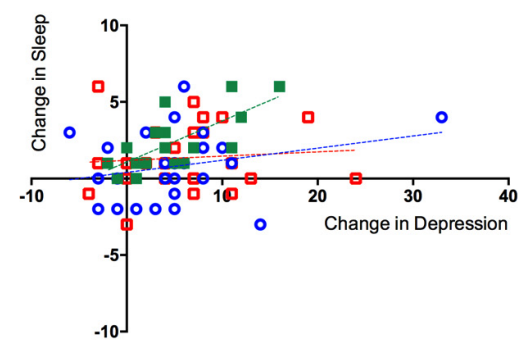

B

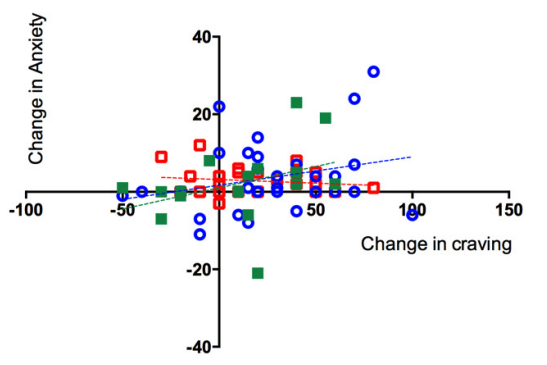

๑- ITBS-L

- CTBS-L

o. cTBS-R

$r=-0.15, p=0.462$

$r=0.371, p=0.129$

$r=0.268, p=0.152$ $\because$ iTBS-L

$\rightarrow$ CTBS-L

- . $C T B S-R$

$r=-0.307, p=0.126$

$r=0.495, p=0.037$

$r=0.156$
D

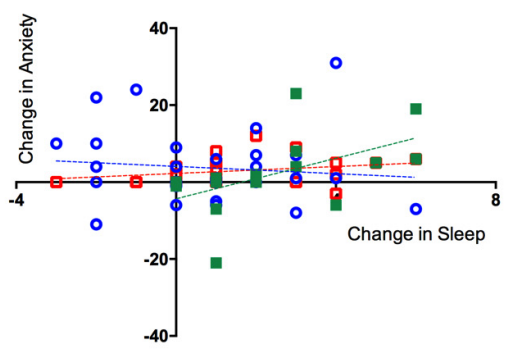

- iTBS-L
- cTBS-L
- cTBS-R
$r=0.278, p=0.68$
$r=0.519, p=0.001^{*}$
$r=-0.105, p=0.168$

F
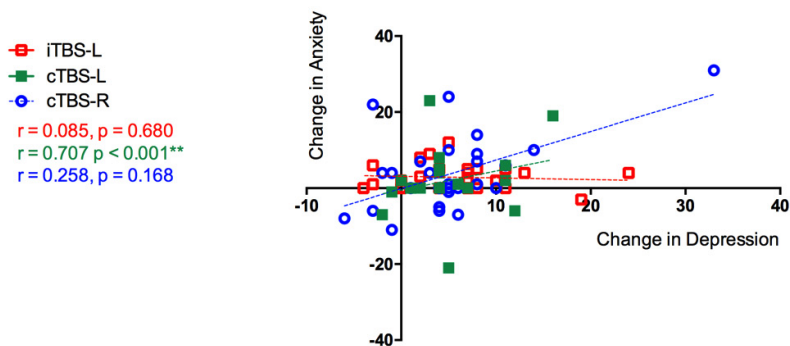

•- ITBS-L

- cTBS-L

- cTBS-R

$r=-0.081, p=0.69$

$r=-0.081, p=0.69$
$r=0.259, p=0.300$

$r=0.544, p=0.002^{\text {** }}$

FIGURE 4 | Correlations between changes between clinical outcomes in three groups. The Pearson correlation between changes in (A) cue-induced craving and sleep quality, (B) cue-induced craving and anxiety level, and (C) cue-induced craving and depression level. (D) Sleep quality and anxiety level and (E) sleep quality and depression level. (F) Anxiety level and depression level. The three treatment groups were assessed separately showing the effects of theta burst stimulation (TBS) between clinical symptoms: intermittent TBS of the left dorsolateral prefrontal cortex (iTBS-L DLPFC) (red), continuous TBS of the left DLPFC (cTBS-L DLPFC) (green), or continuous TBS of the right DLPFC (cTBS-R DLPFC) (blue).

findings suggest that the shorter TBS procedure might serve comparably to other standard rTMS procedures in substance use disorder patients and possibly be relevant dimensionally across a range of clinical symptoms. We emphasize the need for a randomized controlled trial study with a sham control to confirm these findings.

The distribution of the percent change in the primary outcome of cue-elicited craving offered a more fine-grained comparison. We observed the highest percentage of responding, defined as a clinically relevant $50 \%$ change in craving, in the cTBSright DLPFC group. The distributions of all three interventions were not normal, suggesting high interindividual variability to differing TBS protocols. For instance, iTBS-left DLPFC treatment may have two subgroups, with one markedly improving and a second with limited change. As this is a small sample size, further larger studies are required to address these interindividual differences in responses to neuromodulation ( $\mathrm{Li}$ et al., 2015; Suppa et al., 2016).

TBS of the prefrontal cortex might act by enhancing aberrant prefrontal and downstream network function, decreasing aberrant excitability or plasticity or influencing downstream dopaminergic function. Methamphetamine increases synaptic dopamine levels by blocking dopamine reuptake and increasing reverse transport via the dopamine transporter. The chronic use of methamphetamine is associated with impairments in cognition, mood, and sleep (Cruickshank and Dyer, 2009); thus, normalizing mood and sleep symptoms may secondarily improve the secondary consequences of long-term amphetamine use. Chronic psychostimulants are associated with prefrontal hypofunction with impairments related to DLPFC function, 
A

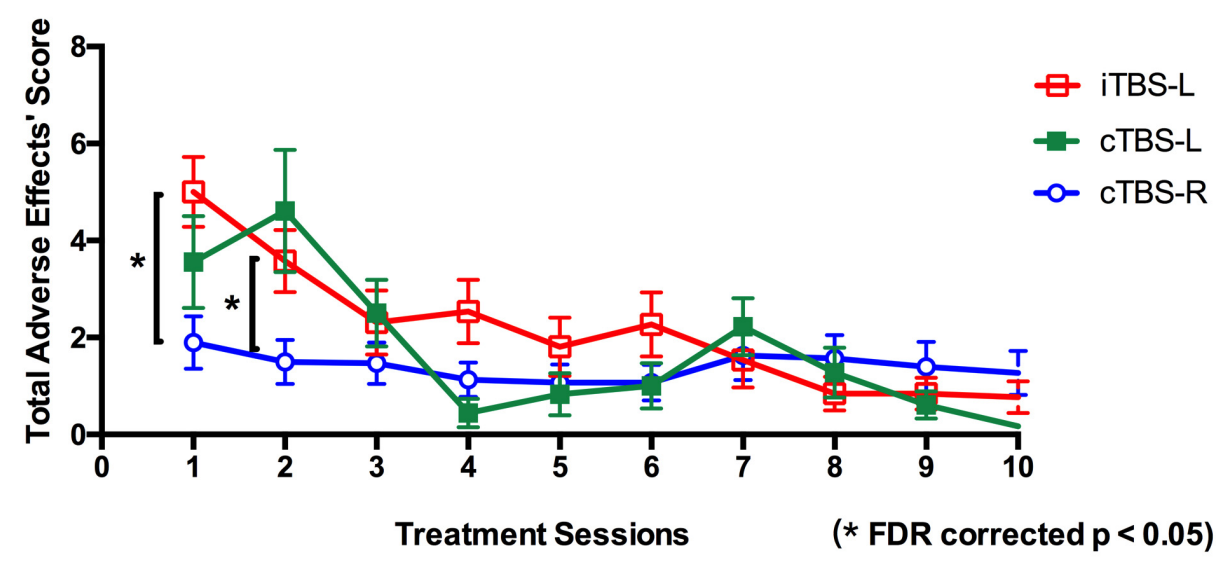

B

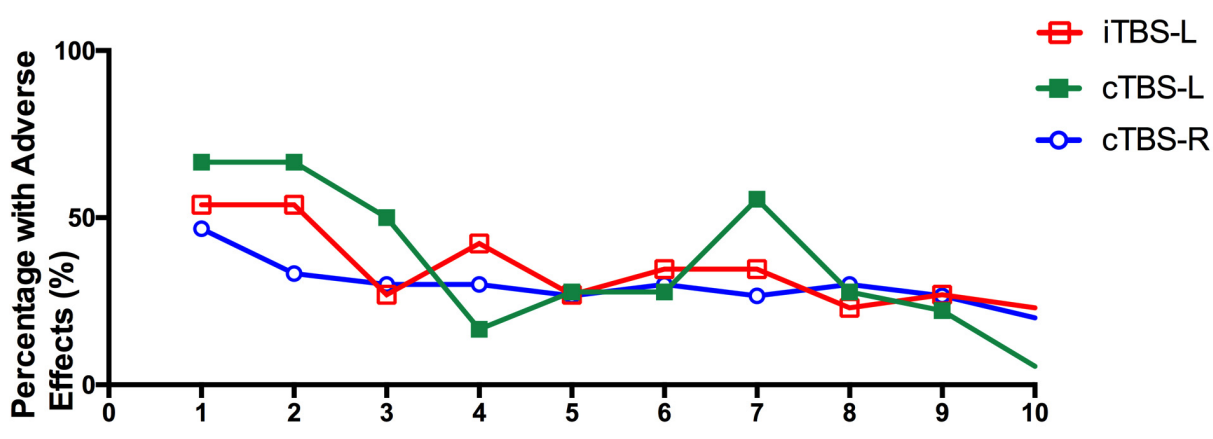

Treatment Sessions

FIGURE 5 | Self-reported adverse effects of twice-daily theta burst stimulation over 10 sessions. (A) Mean total scores of adverse effects (headache, neck pain, scalp pain, tingling, itching, burning sensations, sleepiness, trouble concentrating, and acute mood change) after each theta burst stimulation (TBS) treatment session (intermittent TBS of the left dorsolateral prefrontal cortex (iTBS-L DLPFC) (red), continuous TBS of the left DLPFC (cTBS-L DLPFC) (green), or continuous TBS of the right DLPFC (cTBS-R DLPFC) (blue). Multiple comparisons were corrected false discovery rate (FDR) correction ( ${ }^{*}<<0.05$; error bars denote SEM). (B) Percentage with adverse effects across all participants after each treatment session (red, iTBS-L DLPFC; green, cTBS-L DLPFC; blue, cTBS-R DLPFC).

TABLE 3 | Scores of adverse reactions.

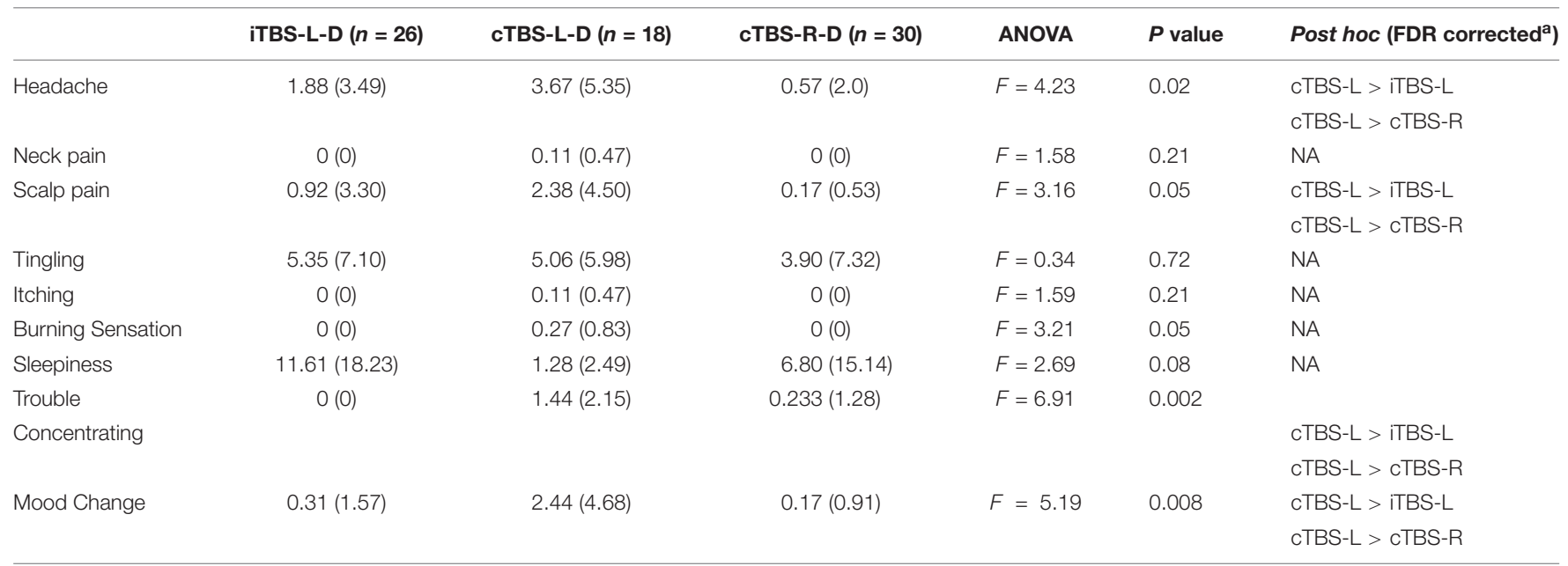

Data are presented as mean (SD). ${ }^{a}$ Multiple comparisons were corrected by the false discovery rate (FDR) correction. 
including executive deficits such as working memory, planning, and goal-directed control (Goldstein and Volkow, 2011; Voon et al., 2015). TBS might thus improve DLPFC function and its associated fronto-striatal network. TMS of the DLPFC paired with functional imaging has shown a decrease in orbitofrontal activity associated nicotine cue-induced craving, particularly when the cue was immediately available, thus implicating a role in intertemporal discounting (Hayashi et al., 2013). Psychostimulants are linked to long-term downregulation of dopaminergic neurotransmission with lower D2/3 receptor levels and blunted dopamine release to psychostimulants (Volkow et al., 2004; Koob and Volkow, 2010). Methamphetamine, in particular, is associated with lower dopamine transporter levels, which can improve with abstinence (Volkow et al., 2001). The downstream influence of TMS to the DLPFC affects caudate synaptic dopamine release in healthy controls (Strafella et al., 2001) and thus may play a role in normalizing methamphetamine-related aberrant dopaminergic function. Further studies are required to assess the underlying mechanisms.

The original TBS neurophysiological study employed $80 \%$ active MT targeting the primary motor cortex (Huang et al., 2005), while other clinical trials have also tried $120 \%$ resting MT of the DLPFC demonstrating both safety and tolerability (Blumberger et al., 2018). Our previous study has shown that TBS (single session/80\% motor threshold) over the motor cortex cannot induce cerebral plasticity, which indicates that neuroplasticity is supposed to be altered in methamphetamine users (Huang et al., 2017). The TBS-related plasticity and behavioral change might highly depend on the intensity, the total number of pulses, the number of sessions, and the stimulating site. In the present study, we adopted 70\% resting MT intensity at DLPFC and show in this pilot study that this threshold is both effective and tolerable for most subjects. The percentage of selfreported adverse events across the sessions reduced from $>50$ to $\sim 20 \%$ within the different groups, suggesting enhanced tolerance and adaptation with repeated TBS. We show that a lower intensity (70\% resting MT) for TBS might still be effective and perhaps enhance tolerability.

Treatment outcomes appeared to be differentially modulated among the three groups. Notably, the decreases in depression score were moderate $(40-50 \%)$ across all protocols, suggesting a potential clinically significant response. We did not observe changes in impulsivity as measured using questionnaires. Previous studies have reported that cTBS but not iTBS of the right DLPFC reduced impulsive choice as measured using the delaydiscounting task in healthy subjects and pathological gambling subjects (Cho et al., 2010; Zack et al., 2016).

Several limitations should be considered. This study focused on the effects of craving to drug cues without long-term follow-up to assess the duration of effect and impact on clinically valid outcomes such as relapse rate or the relationship to natural rewards. Whether fewer or more sessions (e.g., 8 sessions over 4 days or 20 sessions as compared to 10 sessions), a shorter interval between sessions, or a greater number of sessions per day may have a different effect remains to be investigated. The use of placebo or sham TMS in larger sample sizes would be of utility for comparison purposes, although issues have also been highlighted with the use of other forms of control groups (Davis et al., 2013). We note the larger number of dropouts in the cTBSL DLPFC condition, thus limiting its utility as an active control; indeed, if fewer subjects had dropped out, we may have demonstrated the critical group main effect and interaction effect. Moreover, the use of neurophysiological or neuroimaging modalities would also be indicated to explore underlying mechanisms and differences underlying interindividual differences or for outcome prediction (Hawco et al., 2018). We applied iTBS over the left DLPFC but neither the right DLPFC nor a wait-list group due to technical reasons, including the limited number of methamphetaminedependent subjects who could be recruited and the length of the experiment. The clinical effects of iTBS over the right DLPFC and a wait-list group in methamphetamine-dependent subjects should be further investigated. Finally, given that patients were recruited from an ongoing rehabilitation center training program, the findings should be interpreted with caution since daily physical exercise and supportive therapy or individual psychological therapy might alter sleep or mood status. However, crucially, subjects across all groups experienced the same non-TBS-related interventions, and we further show that the improvements in mood and sleep were unrelated to the improvement in craving.

\section{CONCLUSION}

Our findings add to the growing evidence that accelerated TBS might be an efficacious method for craving, mood, sleep, and anxiety symptoms and tolerability in abstinent methamphetamine-dependent subjects. Further larger randomized studies with placebo control and comparisons with standard TBS or standard rTMS protocols are indicated. Critically, our results suggest that the use of both TBS and an accelerated design might show efficacy in targeting methamphetamine craving and emphasize efficiency, potentially facilitating the number of patients that can be treated with each TMS machine and shortening the duration of treatment from several weeks to 1 week.

\section{DATA AVAILABILITY STATEMENT}

The datasets generated for this study are available on request to the corresponding author.

\section{ETHICS STATEMENT}

The studies involving human participants were reviewed and approved by the research ethics boards of Shanghai Mental Health Center, Nanjing Normal University and the local safety monitoring board (Chinese Clinical Trial Registry number, ChiCTR-INR-17013610). The patients/participants provided their written informed consent to participate in this study. 


\section{AUTHOR CONTRIBUTIONS}

T-FY and VV conceptualized and designed the study. DZ, YL, and TL performed the study. DZ, VV, and T-FY analyzed the results and wrote the manuscript together. All authors have read and approved the final version of the manuscript.

\section{FUNDING}

The study is supported by the National Natural Science Foundation of China $(81822017,31771215$ to T-FY, and 31900765 to DZ), Shanghai Sailing Program (19YF1441900 to DZ), and China Postdoctoral Science Foundation (2018M640405 to $\mathrm{DZ}) . \mathrm{VV}$ is supported by the Medical Research Council

\section{REFERENCES}

Amiaz, R., Levy, D., Vainiger, D., Grunhaus, L., and Zangen, A. (2009). Repeated high-frequency transcranial magnetic stimulation over the dorsolateral prefrontal cortex reduces cigarette craving and consumption. Addiction 104, 653-660. doi: 10.1111/j.1360-0443.2008.02448.x

Bakker, N., Shahab, S., Giacobbe, P., Blumberger, D. M., Daskalakis, Z. J., Kennedy, S. H., et al. (2015). Rtms of the dorsomedial prefrontal cortex for major depression: safety, tolerability, effectiveness, and outcome predictors for 10 $\mathrm{hz}$ versus intermittent theta-burst stimulation. Brain Stimul. 8, 208-215. doi: 10.1016/j.brs.2014.11.002

Beck, A. T., Epstein, N., Brown, G., and Steer, R. A. (1988). An inventory for measuring clinical anxiety: psychometric properties. J. Consult. Clin. Psychol. 56, 893-897. doi: 10.1037/0022-006x.56.6.893

Beck, A. T., Ward, C. H., Mendelson, M., Mock, J., and Erbaugh, J. (1961). An inventory for measuring depression. Arch. Gen. Psychiatry 4, 561-571.

Benjamini, Y., and Yekutieli, D. (2001). The control of the false discovery rate in multiple testing under dependency. Annals Stat. 29, 1165-1188. doi: 10.1186/ 1471-2105-9-114

Blumberger, D. M., Vila-Rodriguez, F., Thorpe, K. E., Feffer, K., Noda, Y., Giacoble, P., et al. (2018). Effectiveness of theta burst versus high-frequency repetitive transcranial magnetic stimulation in patients with depression (three-d): a randomised non-inferiority trial. Lancet 391, 1683-1692. doi: 10.1016/S01406736(18)30295-2

Buysse, D. J., Reynolds, C. F. III, Monk, T. H., Berman, S. R., and Kupfer, D. J. (1989). The pittsburgh sleep quality index: a new instrument for psychiatric practice and research. Psychiatry Res. 28, 193-213. doi: 10.1016/0165-1781(89) 90047-4

Camprodon, J. A., Martínez-Raga, J., Alonso-Alonso, M., Shih, M.-C., and PascualLeone, A. (2007). One session of high frequency repetitive transcranial magnetic stimulation (rtms) to the right prefrontal cortex transiently reduces cocaine craving. Drug Alcohol Depend. 86, 91-94. doi: 10.1016/j.drugalcdep.2006. 06.002

Cho, S. S., Ko, J. H., Pellecchia, G., Van Eimeren, T., Cilia, R., and Strafella, A. P. (2010). Continuous theta burst stimulation of right dorsolateral prefrontal cortex induces changes in impulsivity level. Brain Stimul. 3, 170-176. doi: 10.1016/j.brs.2009.10.002

Cruickshank, C. C., and Dyer, K. R. (2009). A review of the clinical pharmacology of methamphetamine. Addiction 104, 1085-1099. doi: 10.1111/j.1360-0443.2009. 02564.x

Davis, N. J., Gold, E., Pascual-Leone, A., and Bracewell, R. M. (2013). Challenges of proper placebo control for non-invasive brain stimulation in clinical and experimental applications. Eur. J. Neurosci. 38, 2973-2977. doi: 10.1111/ejn. 12307

Di Lazzaro, V., Pilato, F., Saturno, E., Oliviero, A., Dileone, M., Mazzone, P., et al. (2005). Theta-burst repetitive transcranial magnetic stimulation suppresses specific excitatory circuits in the human motor cortex. J. Physiol. 565, 945-950. doi: $10.1113 /$ jphysiol.2005.087288
Senior Clinical Fellowship (MR/P008747/1 to VV). T-FY is also sponsored by The Science and Technology Commission of Shanghai Municipality (18QA1403700, 18JC1420304, and 18140901700), Shanghai Municipal Education CommissionGaofeng Clinical Medicine Grant Support (20181715), and Hundred-Talent Fund from Shanghai Municipal Commission of Health (2018BR21). YL is supported by the Key Project of Jiangsu Provincial Department of Science and Technology (BE2017007-5).

\section{ACKNOWLEDGMENTS}

We thank Chengwei Wan for assistance with data collection and management.

Diana, M., Raij, T., Melis, M., Nummenmaa, A., Leggio, L., and Bonci, A. (2017) Rehabilitating the addicted brain with transcranial magnetic stimulation. Nat. Rev. Neurosci. 18, 685-693. doi: 10.1038/nrn.2017.113

Dinur-Klein, L., Dannon, P., Hadar, A., Rosenberg, O., Roth, Y., Kotler, M., et al. (2014). Smoking cessation induced by deep repetitive transcranial magnetic stimulation of the prefrontal and insular cortices: A prospective, randomized controlled trial. Biol. Psychiatry 76, 742-749. doi: 10.1016/j.biopsych.2014. 05.020

Everitt, B. J., and Robbins, T. W. (2016). Drug addiction: Updating actions to habits to compulsions ten years on. Annu. Rev. Psychol. 67, 23-50. doi: 10.1146/ annurev-psych-122414-033457

George, M. S., Stallings, L. E., Speer, A. M., Nahas, Z., Spicer, K. M., Vincent, D. J., et al. (1999). Prefrontal repetitive transcranial magnetic stimulation (rtms) changes relative perfusion locally and remotely. Human Psychopharmacol. Clin. Exp. 14, 161-170. doi: 10.1002/(sici)1099-1077(199904)14:3<161::aid-hup73> 3.0.co;2-2

Goldstein, R. Z., and Volkow, N. D. (2011). Dysfunction of the prefrontal cortex in addiction: neuroimaging findings and clinical implications. Nat. Rev. Neurosci. 12, 652-669. doi: 10.1038/nrn3119

Gordon, H. W. (2016). Laterality of brain activation for risk factors of addiction. Curr. Drug Abuse Rev. 9, 1-18. doi: 10.2174/1874473709666151217121309

Hanlon, C. A., Kearney-Ramos, T., Dowdle, L. T., Hamilton, S., DeVries, W., Mithoefer, O., et al. (2017). Developing repetitive transcranial magnetic stimulation (rtms) as a treatment tool for cocaine use disorder: a series of six translational studies. Curr. Behav. Neurosci. Rep. 4, 341-352. doi: 10.1007/ s40473-017-0135-4

Hawco, C., Voineskos, A. N., Steeves, J. K. E., Dickie, E. W., Viviano, J. D., Downar, J., et al. (2018). Spread of activity following tms is related to intrinsic resting connectivity to the salience network: A concurrent tms-fmri study. Cortex 108, 160-172. doi: 10.1016/j.cortex.2018.07.010

Hayashi, T., Ko, J. H., Strafella, A. P., and Dagher, A. (2013). Dorsolateral prefrontal and orbitofrontal cortex interactions during self-control of cigarette craving. Proc. Natl. Acad. Sci. U.S.A. 110, 4422-4427. doi: 10.1073/pnas.121218 5110

Herwig, U., Satrapi, P., and Schönfeldt-Lecuona, C. (2003). Using the international 10-20 eeg system for positioning of transcranial magnetic stimulation. Brain Topogr. 16, 95-99. doi: 10.1023/B:BRAT.0000006333.93597.9d

Höppner, J., Broese, T., Wendler, L., Berger, C., and Thome, J. (2011). Repetitive transcranial magnetic stimulation (rtms) for treatment of alcohol dependence. World J. Biol. Psychiatry 12, 57-62.

Huang, X., Chen, Y.-Y., Shen, Y., Cao, X., Li, A., Liu, Q., et al. (2017). Methamphetamine abuse impairs motor cortical plasticity and function. Mol. Psychiatry 22, 1274-1281. doi: 10.1038/mp.2017.143

Huang, Y.-Z., Edwards, M. J., Rounis, E., Bhatia, K. P., and Rothwell, J. C. (2005). Theta burst stimulation of the human motor cortex. Neuron 45, 201-206.

Kammer, T., Beck, S., Thielscher, A., Laubis-Herrmann, U., and Topka, H. (2001). Motor thresholds in humans: a transcranial magnetic stimulation study 
comparing different pulse waveforms, current directions and stimulator types. Clin. Neurophysiol. 112, 250-258. doi: 10.1016/S1388-2457(00)00513-7

Koob, G. F., and Volkow, N. D. (2010). Neurocircuitry of addiction. Neuropsychopharmacology 35:217.

Li, L. M., Uehara, K., and Hanakawa, T. (2015). The contribution of interindividual factors to variability of response in transcranial direct current stimulation studies. Front. Cell. Neurosci. 9:181. doi: 10.3389/fncel.2015.00181

Li, X., Hartwell, K. J., Owens, M., Lematty, T., Borckardt, J. J., Hanlon, C. A., et al. (2013a). Repetitive transcranial magnetic stimulation of the dorsolateral prefrontal cortex reduces nicotine cue craving. Biol. Psychiatry 73, 714-720. doi: 10.1016/j.biopsych.2013.01.003

Li, X., Malcolm, R. J., Huebner, K., Hanlon, C. A., Taylor, J. J., Brady, K. T., et al. (2013b). Low frequency repetitive transcranial magnetic stimulation of the left dorsolateral prefrontal cortex transiently increases cue-induced craving for methamphetamine: a preliminary study. Drug Alcohol Depend. 133, 641-646. doi: 10.1016/j.drugalcdep.2013.08.012

Lopez-Alonso, V., Cheeran, B., Rio-Rodriguez, D., and Fernandez-Del-Olmo, M. (2014). Inter-individual variability in response to non-invasive brain stimulation paradigms. Brain Stimul. 7, 372-380. doi: 10.1016/j.brs.2014.02.004

McGirr, A., Van den Eynde, F., Tovar-Perdomo, S., Fleck, M. P. A., and Berlim, M. T. (2015). Effectiveness and acceptability of accelerated repetitive transcranial magnetic stimulation (rtms) for treatment-resistant major depressive disorder: An open label trial. J. Affect. Disord. 173, 216-220. doi: 10.1016/j.jad.2014.10.068

Mishra, B. R., Nizamie, S. H., Das, B., and Praharaj, S. K. (2010). Efficacy of repetitive transcranial magnetic stimulation in alcohol dependence: a sham-controlled study. Addiction 105, 49-55. doi: 10.1111/j.1360-0443.2009. 02777.x

Modirrousta, M., Meek, B. P., and Wikstrom, S. L. (2018). Efficacy of twice-daily vs once-daily sessions of repetitive transcranial magnetic stimulation in the treatment of major depressive disorder: a retrospective study. Neuropsychiatr. Dis. Treat 14, 309-316. doi: 10.2147/NDT.S151841

Politi, E., Fauci, E., Santoro, A., and Smeraldi, E. (2008). Daily sessions of transcranial magnetic stimulation to the left prefrontal cortex gradually reduce cocaine craving. Am. J. Addict. 17, 345-346. doi: 10.1080/10550490802139283

Reise, S. P., Moore, T. M., Sabb, F. W., Brown, A. K., and London, E. D. (2013). The barratt impulsiveness scale-11: reassessment of its structure in a community sample. Psychol. Assess. 25, 631-642. doi: 10.1037/a0032161

Rose, J. E., McClernon, F. J., Froeliger, B., Behm, F. M., Preud'homme, X., and Krystal, A. D. (2011). Repetitive transcranial magnetic stimulation of the superior frontal gyrus modulates craving for cigarettes. Biol. Psychiatry 70, 794-799. doi: 10.1016/j.biopsych.2011.05.031

Sanna, A., Fattore, L., Badas, P., Corona, G., Cocco, V., and Diana, M. (2019). Intermittent theta burst stimulation of the prefrontal cortex in cocaine use disorder: a pilot study. Front. Neurosci. 13:765. doi: 10.3389/fnins.2019.00765

Schulze, L., Feffer, K., Lozano, C., Giacobbe, P., Daskalakis, Z. J., Blumberger, D. M., et al. (2018). Number of pulses or number of sessions? an open-label study of trajectories of improvement for once-vs. Twice-daily dorsomedial prefrontal rtms in major depression. Brain Stimul. 11, 327-336. doi: 10.1016/j.brs.2017. 11.002

Sheffer, C. E., Bickel, W. K., Brandon, T. H., Franck, C. T., Deen, D., Panissidi, L., et al. (2018). Preventing relapse to smoking with transcranial magnetic stimulation: feasibility and potential efficacy. Drug Alcohol Depend. 182, 8-18. doi: 10.1016/j.drugalcdep.2017.09.037

Shen, Y., Cao, X., Tan, T., Shan, C., Wang, Y., Pan, J., et al. (2016). 10-hz repetitive transcranial magnetic stimulation of the left dorsolateral prefrontal cortex reduces heroin cue craving in long-term addicts. Biol. Psychiatry 80, e13-e14. doi: 10.1016/j.biopsych.2016.02.006

Steele, V., Maxwell, A. M., Ross, T., Stein, E., and Salmeron, B. J. (2019). Accelerated intermittent theta-burst stimulation as a treatment for cocaine use disorder: a proof-of-concept study. Front. Neurosci. 13:1147. doi: 10.3389/fnins.2019. 01147

Strafella, A. P., Paus, T., Barrett, J., and Dagher, A. (2001). Repetitive transcranial magnetic stimulation of the human prefrontal cortex induces dopamine release in the caudate nucleus. J. Neurosci. 21, RC157-RC157.

Su, H., Zhong, N., Gan, H., Wang, J., Han, H., Chen, T., et al. (2017). High frequency repetitive transcranial magnetic stimulation of the left dorsolateral prefrontal cortex for methamphetamine use disorders: a randomised clinical trial. Drug Alcohol Depend. 175, 84-91. doi: 10.1016/j.drugalcdep.2017. 01.037

Suppa, A., Huang, Y. Z., Funke, K., Ridding, M. C., Cheeran, B., Di Lazzaro, V., et al. (2016). Ten years of theta burst stimulation in humans: established knowledge, unknowns and prospects. Brain Stimul. 9, 323-335. doi: 10.1016/j. brs.2016.01.006

Terraneo, A., Leggio, L., Saladini, M., Ermani, M., Bonci, A., and Gallimberti, L. (2016). Transcranial magnetic stimulation of dorsolateral prefrontal cortex reduces cocaine use: a pilot study. Eur. Neuropsychopharmacol. 26, 37-44. doi: 10.1016/j.euroneuro.2015.11.011

Volkow, N. D., Chang, L., Wang, G.-J., Fowler, J. S., Franceschi, D., Sedler, M., et al. (2001). Loss of dopamine transporters in methamphetamine abusers recovers with protracted abstinence. J. Neurosci. 21, 9414-9418. doi: 10.1523/jneurosci. 21-23-09414.2001

Volkow, N. D., Fowler, J. S., Wang, G.-J., and Swanson, J. M. (2004). Dopamine in drug abuse and addiction: results from imaging studies and treatment implications. Mol. Psychiatry 9:557. doi: 10.1038/sj.mp.4001507

Voon, V., Derbyshire, K., Rück, C., Irvine, M. A., Worbe, Y., Enander, J., et al. (2015). Disorders of compulsivity: a common bias towards learning habits. Mol. Psychiatry 20:345. doi: 10.1038/mp.2014.44

Yavari, F., Shahbabaie, A., Leite, J., Carvalho, S., Ekhtiari, H., and Fregni, F. (2016). Noninvasive brain stimulation for addiction medicine: from monitoring to modulation. Prog. Brain Res. 224, 371-399. doi: 10.1016/bs.pbr.2015. 08.007

Zack, M., Cho, S. S., Parlee, J., Jacobs, M., Li, C., Boileau, I., et al. (2016). Effects of high frequency repeated transcranial magnetic stimulation and continuous theta burst stimulation on gambling reinforcement, delay discounting, and stroop interference in men with pathological gambling. Brain Stimul. 9, 867875. doi: 10.1016/j.brs.2016.06.003

Zhang, J. J., Fong, K. N., Ouyang, R. G., Siu, A. M., and Kranz, G. S. (2019). Effects of repetitive transcranial magnetic stimulation (rtms) on craving and substance consumption in patients with substance dependence: a systematic review and meta-analysis. Addiction 114, 2137-2149. doi: 10.1111/add.14753

Conflict of Interest: The authors declare that the research was conducted in the absence of any commercial or financial relationships that could be construed as a potential conflict of interest.

Copyright () 2020 Zhao, Li, Liu, Voon and Yuan. This is an open-access article distributed under the terms of the Creative Commons Attribution License (CC BY). The use, distribution or reproduction in other forums is permitted, provided the original author(s) and the copyright owner(s) are credited and that the original publication in this journal is cited, in accordance with accepted academic practice. No use, distribution or reproduction is permitted which does not comply with these terms. 